\title{
SPECTRAL ZETA FUNCTIONS FOR COMPACT SYMMETRIC SPACES OF RANK ONE
}

\author{
AKIRA IKEDA*
}

\begin{abstract}
We study the spectral zeta function $\zeta_{M}(s)$ associated with the spectrum of Laplacian acting on functions of a compact simply connected Riemannian symmetric space $M$ of rank one and the spectral zeta function $\zeta_{S^{n}}^{p . \delta}(s)$ associated with the spectrum of Laplacian acting on $p$-forms of the sphere $S^{n} \quad$ We give the residues of $\zeta_{M}(s)$ and $\zeta_{S^{n}}^{p}(s)$ explictly. For the odd dimensional sphere $S^{n}$, we show that $\zeta_{S^{n}}^{p, \delta}(s)$ vanishes at negative integers.
\end{abstract}

\section{Introduction}

Let $M$ be a compact connected Riemannian manifold, $\Delta_{M}$ the LaplaceBeltram operator acting on smooth functions. It has a discrete specrtrum

$$
0=\lambda_{0}<\lambda_{1} \leq \lambda_{2} \leq \cdots \leq \lambda_{n} \leq \cdots,
$$

where every $\lambda_{l}$ is repeated with its multiplicity. The spectral zeta function of $M$ which is well defined in $\operatorname{Re}(s)>\operatorname{dim} M / 2$ is given by

$$
\zeta_{M}(s)=\sum_{k=1}^{\infty} \lambda_{k}^{-s}
$$

In [9] Minakshisundaram-Pleijel proved $\zeta_{M}(s)$ has a meromorphic continuation on the whole complex plane $\boldsymbol{C}$ and analytic on $\boldsymbol{C}$ except at simple poles at $s=$ $\operatorname{dim} M / 2-k(k=0,1,2, \ldots)$, and express the residues in terms of metric invariants of $M$. In [3], [4] Carletti and Monti Bragardin studied Dirichlet series $L(s)=\sum_{k=1}^{\infty} P(k) /\left(\left(k+d_{1}\right)^{2}\left(k+d_{2}\right)^{s}\right)$ and applied to the spectral zeta functions for the standard spheres of constant curvature 1. They give the residues of the spectral zeta functions explicitly.

Let $\Delta_{M}^{p}$ be the Laplace-Beltrami operator acting on smooth $p$-forms $\Lambda^{p}(M)$. Denote the differential and codifferential of $M$ by $d$ and $\delta$, respectively;

$$
\begin{aligned}
& d: \Lambda^{p}(M) \rightarrow \Lambda^{p+1}(M), \\
& \delta: \Lambda^{p}(M) \rightarrow \Lambda^{p-1}(M) .
\end{aligned}
$$

\footnotetext{
* The author was partially supported by Grants-1n-A1d for Scientific Research (No. 09640111), the Ministry of Education, Sciences and Culture, Japan.

Received July 21, 1999; revised March 7, 2000.
} 
Let $H^{p}(M)$ be the space of harmonic $p$-forms. We define the space of $\delta$ closed forms by

$$
\Lambda_{\delta}^{p}(M)=\left\{\alpha \in \Lambda^{p}(M) \mid \delta \alpha=0, \alpha \perp H^{p}(M)\right\},
$$

and the space of $d$-closed forms by

$$
\Lambda_{d}^{p}(M)=\left\{\alpha \in \Lambda^{p}(M) \mid d \alpha=0, \alpha \perp H^{p}(M)\right\} .
$$

Since $d$ and $\delta$ commute with $\Delta_{M}^{p}, \Delta_{M}^{p}$ acts invariantly on $\Lambda_{\delta}^{p}(M)$. We say the eigenvalue $\lambda$ to be $\delta$-eigenvalue if there is an eigen $p$-form in $\Lambda_{\delta}^{p}(M)$ with the eigenvalue $\lambda$.

Let

$$
0<\lambda_{1}^{p} \leq \lambda_{2}^{p} \leq \cdots \leq \lambda_{n}^{p} \leq \cdots,
$$

be the set of all the $\delta$-eigenvalues, where every $\lambda_{l}^{p}$ is repeated with its multiplicity. We define the spectral zeta function $\zeta_{M}^{p, \delta}(s)$ by

$$
\zeta_{M}^{p, \delta}(s)=\sum_{i=1}^{\infty}\left(\lambda_{i}^{p}\right)^{-s}
$$

Note that

$$
\zeta_{M}^{0, \delta}(s)=\zeta_{M}(s)
$$

In this paper, we study the spectral zeta function $\zeta_{S^{n}}^{p, \delta}(s)$ of the standard spheres of constant curvature 1 and $\zeta_{M}(s)$ of other compact simply connected Riemannian symmetric spaces of rank 1 . We give the residues of their spectral zeta functions explicitly which have much simpler forms than Carletti and Monti Bragardin's results.

It is well know that the Riemann zeta function has trivial zeros at any negative even integer. In [10] Minakshisundaram proved that the spectral zeta function $\zeta_{S^{n}}(s)$ of the odd dimensional sphere vanishes at any negative integer. In the last section, we show the spectral zeta function $\zeta_{S^{n}}^{p, \delta}(s)$ of the odd dimensional sphere also vanishes at any negative integer.

\section{Dirichlet series $L(s)=\sum_{k=1}^{\infty} P(k) /\left(\left(k+d_{1}\right)^{s}\left(k+d_{2}\right)^{s}\right)$}

Let $P(x)$ be a polynomial of degree $N$. For two real numbers $d_{1}$ and $d_{2}$ with $d_{2} \geq d_{1}>-1$, we consider the Dirichlet series

$$
L(s)=\sum_{k=1}^{\infty} \frac{P(k)}{\left(k+d_{1}\right)^{s}\left(k+d_{2}\right)^{s}} .
$$

In this section, we review Carletti-Monti Bragardin's results for $L(s)$. We prepare some notations for later use. Let $P_{m-j}^{m}(\beta)$ be polynomials in $\beta$ of degree $m-j$ defined by 


$$
\begin{aligned}
& P_{0}^{0}(\beta)=1, \quad P_{m}^{m}(\beta)=\frac{1}{m !} \prod_{k=1}^{m}(\beta-k), \\
& P_{m-j}^{m}(\beta)=\frac{1}{j !} \frac{d^{J}}{d \beta^{J}} P_{m}^{m}(\beta) \quad 0 \leq j \leq m .
\end{aligned}
$$

Put

$$
P(x)=\sum_{j=0}^{N} a_{j} x^{J},
$$

and define the constants $a_{p}^{m},(m, p \geq 1)$ by

$$
a_{p}^{m}=\sum_{\ell=1}^{p}(-1)^{p-\ell}\left(\begin{array}{c}
m+1 \\
p-\ell
\end{array}\right) \ell^{m} .
$$

For $a>0$, the Hurewicz zeta function is defined by

$$
\zeta(s, a)=\sum_{r=0}^{\infty} \frac{1}{(r+a)^{s}} .
$$

Note that the Riemann zeta function $\zeta(s)$ is

$$
\zeta(s)=\zeta(s, 1) .
$$

Then we have the following formula (see [3]):

Proposition 1.

$$
\begin{aligned}
L(s)= & \sum_{\ell=0}^{\infty}\left(\frac{d_{2}-d_{1}}{2}\right)^{2 \ell}(-1)^{\ell}\left(\begin{array}{c}
-s \\
\ell
\end{array}\right) \\
& \times\left[a_{0} \zeta\left(2 s+2 \ell, 1+\frac{d_{1}+d_{2}}{2}\right)\right. \\
& \left.+\sum_{m=1}^{N} \sum_{j=0}^{m} \sum_{l=1}^{m} a_{m} a_{i}^{m} P_{m-\jmath}^{m}\left(m+1-i-\frac{d_{1}+d_{2}}{2}\right) \zeta\left(2 s+2 \ell-j, 1+\frac{d_{1}+d_{2}}{2}\right)\right] .
\end{aligned}
$$

Note that if $d_{1}=d_{2}$, then the above formula shoud be read as follows;

$$
\begin{aligned}
L(s)= & a_{0} \zeta\left(2 s, 1+d_{1}\right) \\
& +\sum_{m=1}^{N} \sum_{j=0}^{m} \sum_{l=1}^{m} a_{m} a_{i}^{m} P_{m-j}^{m}\left(m+1-i-d_{1}\right) \zeta\left(2 s-j, 1+d_{1}\right) .
\end{aligned}
$$

From this formula, we can get easily the following corollary: 
Corollary 1. 1. If $P(X)=1$, then

$$
L(s)=\sum_{\ell=0}^{\infty}\left(\frac{d_{2}-d_{1}}{2}\right)^{2 \ell}(-1)^{\ell}\left(\begin{array}{c}
-s \\
\ell
\end{array}\right) \zeta\left(2 s+2 \ell, 1+\frac{d_{2}-d_{1}}{2}\right) .
$$

2. If $P(X)=X+\left(d_{2}-d_{1}\right) / 2$, then

$$
L(s)=\sum_{\ell=0}^{\infty}\left(\frac{d_{2}-d_{1}}{2}\right)^{2 \ell}(-1)^{\ell}\left(\begin{array}{c}
-s \\
\ell
\end{array}\right) \zeta\left(2 s+2 \ell-1,1+\frac{d_{2}-d_{1}}{2}\right) .
$$
follows

In the above Corollary, if $d_{1}=d_{2}$, then this formula should be read as

$$
L(s)=\zeta\left(2 s, 1+d_{1}\right)
$$

and

$$
L(s)=\zeta\left(2 s-1,1+d_{1}\right),
$$

according to $P(X)=1$ or $P(X)=X+d_{1}$.

\section{Spectral zeta functions for spheres $S^{n}$}

Let $S^{n}$ denote the $n$-dimensional standard sphere of constant curvature 1 . By [7], all the positive $\delta$-eigenvalues of $\Delta_{S^{n}}^{p}(0 \leq p \leq n / 2)$ are

$$
(k+p)(k+n-p-1), \quad k \geq 1,
$$

with their multiplicities

$$
\frac{(2 k+n-1) k(k+1)(k+2) \cdots(k+n-1)}{p !(n-p-1) !(k+p)(k+n-p-1)} .
$$

The spectral zeta function $\zeta_{S^{n}}^{p, \delta}(s)$ are given as follows;

$$
\zeta_{S^{n}}^{p, \delta}(s)=\frac{2}{p !(n-p-1) !} \sum_{k=1}^{\infty} \frac{(k+m) k(k+1)(k+2) \cdots(k+n-1)}{((k+p)(k+n-p-1))^{s+1}}
$$

We treat with $\zeta_{S^{n}}^{p, \delta}(s)$ separately according to whether $n$ is odd or even.

$S^{n}, n$ odd $(n=2 m+1)$.

Define the polynomial $F_{n, p}(x)$ by

$$
\begin{aligned}
F_{n, p}(x) & =\frac{2 \prod_{l=0}^{m}\left(x+(m-p)^{2}-i^{2}\right)}{p !(n-p-1) !} \\
& =\sum_{J=1}^{m+1} \alpha_{n, p, j} x^{J} .
\end{aligned}
$$

Then we have 


$$
\begin{aligned}
\zeta_{S^{n}}^{p, \delta}(s) & =\sum_{k=1}^{\infty} \frac{F_{n, p}((k+p)(k+n-p-1))}{((k+p)(k+n-p-1))^{s+1}} \\
& =\sum_{j=1}^{m+1} \alpha_{n, p, j} \sum_{k=1}^{\infty} \frac{1}{((k+p)(k+n-p-1))^{s+1-j}}
\end{aligned}
$$

Using Corollary 1, we have

(21) $\zeta_{S^{n}}^{p, \delta}(s)$

$$
\begin{aligned}
= & \sum_{j=0}^{m+1} \alpha_{n, p, j} \sum_{\ell=0}^{\infty}(m-p)^{2 \ell} \frac{\Gamma(s+1-j+\ell)}{\ell ! \Gamma(s+1-j)} \zeta(2(s+1-j)+2 \ell-1,1+m) \\
= & \sum_{j=1}^{m+1} \alpha_{n, p, j} \sum_{\ell=0}^{\infty}(m-p)^{2 \ell} \frac{\Gamma(s+1-j+\ell)}{\ell ! \Gamma(s+1-j)} \zeta(2 s+2(\ell-j+1), 1+m) \\
= & \sum_{t=0}^{\infty} \sum_{\ell=t}^{m+t} \alpha_{n, p, \ell-t+1}(m-p)^{2 \ell} \frac{\Gamma(s+t)}{\ell ! \Gamma(s+t-\ell)} \zeta(2 s+2 t, 1+m) \\
& +\sum_{t=-(n-1)}^{-1} \sum_{\ell=0}^{n-1+t} \alpha_{n, p, \ell-t+1}(m-p)^{2 \ell} \frac{\Gamma(s+t)}{\ell ! \Gamma(s+t-\ell)} \zeta(2 s+2 t, 1+m) .
\end{aligned}
$$

From this formula. we have

THEOREM 1. The spectral zeta function $\zeta_{S^{n}}^{p, \delta}(s)$ of the odd dimensional standard sphere $S^{2 m+1}$ has a meromorphic continuation on the whole complex plane with at most simple poles at $s=n / 2-k(k=0,1,2, \ldots)$. The residue at $s=n / 2-k$ is given as

$$
\left\{\begin{array}{l}
\frac{1}{2} \sum_{\ell=0}^{k} \alpha_{n, p, \ell+m-k+1}\left(-\frac{(m-p)^{2}}{4}\right)^{\ell}\left(\begin{array}{c}
2 \ell \\
\ell
\end{array}\right) \quad \text { if } m \geq k \geq 0 \\
\frac{1}{2} \sum_{\ell=m-k}^{2 m-k} \alpha_{n, p, \ell-m+k+1}\left(-\frac{(m-p)^{2}}{4}\right)^{\ell}\left(\begin{array}{c}
2 \ell \\
\ell
\end{array}\right) \text { if } k>m
\end{array}\right.
$$

$S^{n}, n$ even $(n=2 m)$.

Define the function $F_{n, p}(x)$ by

$$
\begin{aligned}
F_{n, p}(x) & =\frac{2 \prod_{l=1}^{m}\left(x+((n-1) / 2-p)^{2}-(i-1 / 2)^{2}\right)}{p !(n-p-1) !} \\
& =\sum_{j=1}^{m} \alpha_{n, p, j} x^{J} .
\end{aligned}
$$


Then we have

$$
\begin{aligned}
\zeta_{S^{n}}^{p, \delta}(s) & =\sum_{k=1}^{\infty} \frac{(k+(n-1) / 2) F_{n, p}((k+p)(k+n-p-1))}{((k+p)(k+n-p-1))^{s+1}} \\
& =\sum_{j=1}^{m} \alpha_{n, p, j} \sum_{k=1}^{\infty} \frac{(k+(n-1) / 2)}{((k+p)(k+n-p-1))^{s+1-j}} .
\end{aligned}
$$

Using Corollary 1, we have

$$
\begin{aligned}
\zeta_{S^{n}}^{p, \delta}(s) & \\
= & \sum_{j=1}^{m} \alpha_{n, p, j} \sum_{\ell=0}^{\infty}\left(\frac{n-1}{2}-p\right)^{2 \ell} \frac{\Gamma(s+1-j+\ell)}{\ell ! \Gamma(s+1-j)} \zeta\left(2 s+2(\ell-j+1), 1+\frac{n-1}{2}\right) \\
= & \sum_{t=0}^{\infty} \sum_{\ell=t}^{m+t} \alpha_{n, p, \ell-t+1}\left(\frac{n-1}{2}-p\right)^{2 \ell} \frac{\Gamma(s+t)}{\ell ! \Gamma(s+t-\ell)} \zeta\left(2 s+2 t-1,1+\frac{n-1}{2}\right) \\
& +\sum_{t=-m}^{-1} \sum_{\ell=0}^{m-t} \alpha_{n, p, \ell+t+1}\left(\frac{n-1}{2}-p\right)^{2 \ell} \frac{\Gamma(s+t)}{\ell ! \Gamma(s+t-\ell)} \zeta\left(2 s+2 t-1,1+\frac{n-1}{2}\right) .
\end{aligned}
$$

From this formula, we have

THEOREM 2. The spectral zeta function $\zeta_{S^{n}}^{p, \delta}(s)$ of the even dimensional sphere $S^{2 m}$ has a meromorphic continuation on the whole complex plane with at most simple poles at $s=k(k=1,2, \ldots m)$. The residue at $s=k$ is $\alpha_{n, p, k} / 2$.

\section{Spectral zeta functions for complex projective spaces $P^{n}(C)$}

Let $P^{n}(C)$ denote the complex projective $n$-space with Riemannian metric induced by $(n+1) \times$ negative of the Cartan-Killing form of $S U(n+1)$. Then every geodesic on $P^{n}(C)$ is closed of length $\pi$. By [2], all the eigenvalues of $\Delta_{P^{n}(C)}^{0}$ are given by

$$
k(k+n), \quad k \geq 0,
$$

with their multiplicities

$$
\frac{(2 k+n)((k+1)(k+2) \cdots(k+n-1))^{2}}{n !(n-1) !} .
$$

So that the spectral zeta function $\zeta_{P^{n}(\boldsymbol{C})}(s)$ of $P^{n}(\boldsymbol{C})$ is of the form:

$$
\zeta_{P^{n}(C)}(s)=\frac{2}{n !(n-1) !} \sum_{k=1}^{\infty} \frac{(k+n / 2)\{(k+1)(k+2) \cdots(k+n-1)\}^{2}}{(k(k+n))^{s}} .
$$

We treat with $\zeta_{P^{n}(\boldsymbol{C})}(s)$, separately according to whether $n$ is odd or even. 
$P^{n}(C), n$ odd $(n=2 m+1)$.

Define the polynomial $G_{n}(x)$ by

$$
\begin{aligned}
G_{n}(x) & =\frac{2 \prod_{l=0}^{m}\left(x+(n / 2)^{2}-(i-1 / 2)^{2}\right)^{2}}{n !(n-1) !} \\
& =\sum_{j=0}^{n-1} \gamma_{n, J} x^{J} .
\end{aligned}
$$

Then we have

$$
\begin{aligned}
\zeta_{P^{n}(\boldsymbol{C})}(s) & =\sum_{k=1}^{\infty} \frac{(k+n / 2) G_{n}(k(k+n))}{(k(k+n))^{s}} \\
& =\sum_{j=0}^{n-1} \gamma_{n, j} \sum_{k=1}^{\infty} \frac{(k+n / 2)}{(k(k+n))^{s-\jmath}} .
\end{aligned}
$$

Using Corollary 1, we have

$$
\begin{aligned}
\zeta_{P^{n}(\boldsymbol{C})}(s)= & \sum_{j=0}^{n-1} \gamma_{n, j} \sum_{\ell=0}^{\infty}\left(\frac{n}{2}\right)^{2 \ell} \frac{\Gamma(s-j+\ell)}{\ell ! \Gamma(s-j)} \zeta\left(2(s-j)+2 \ell-1,1+\frac{n}{2}\right) \\
= & \sum_{j=0}^{n-1} \gamma_{n, j} \sum_{\ell=0}^{\infty}\left(\frac{n}{2}\right)^{2 \ell} \frac{\Gamma(s-j+\ell)}{\ell ! \Gamma(s-j)} \zeta\left(2 s+2(\ell-j)-1,1+\frac{n}{2}\right) \\
= & \sum_{t=0}^{\infty} \sum_{\ell=t}^{n-1+t} \gamma_{n, \ell-t}\left(\frac{n}{2}\right)^{2 \ell} \frac{\Gamma(s+t)}{\ell ! \Gamma(s+t-j)} \zeta\left(2 s+2 t-1,1+\frac{n}{2}\right) \\
& +\sum_{t=-(n-1)}^{-1} \sum_{\ell=0}^{n-1+t} \gamma_{n, \ell-t}\left(\frac{n}{2}\right)^{2 \ell} \frac{\Gamma(s+t)}{\ell ! \Gamma(s+t-j)} \zeta\left(2 s+2 t-1,1+\frac{n}{2}\right) .
\end{aligned}
$$

$P^{n}(\boldsymbol{C}), n$ even $(n=2 m)$.

Define the polynomial $G_{n}(x)$ by

$$
\begin{aligned}
G_{n}(x) & =\frac{2\left(x+m^{2}\right) \prod_{l=1}^{m-1}\left(x+m^{2}-i^{2}\right)^{2}}{n !(n-1) !} \\
& =\sum_{J=0}^{n-1} \gamma_{n, j} x^{J} .
\end{aligned}
$$

Then we have

$$
\begin{aligned}
\zeta_{P^{n}(\boldsymbol{C})}(s) & =\sum_{k=1}^{\infty} \frac{(k+m) G_{n}(k(k+n))}{(k(k+n))^{s}} \\
& =\sum_{j=0}^{n-1} \gamma_{n, j} \sum_{k=1}^{\infty} \frac{(k+m)}{(k(k+n))^{s-j}}
\end{aligned}
$$


Using Corollary 1, we have

$$
\begin{aligned}
\zeta_{P^{n}(\boldsymbol{C})}(s)= & \sum_{j=0}^{n-1} \gamma_{n, j} \sum_{\ell=0}^{\infty} m^{2 \ell} \frac{\Gamma(s-j+\ell)}{\ell ! \Gamma(s-j)} \zeta(2(s-j)+2 \ell-1,1+m) \\
= & \sum_{j=0}^{n-1} \gamma_{n, j} \sum_{\ell=0}^{\infty} m^{2 \ell} \frac{\Gamma(s-j+\ell)}{\ell ! \Gamma(s-j)} \zeta(2 s+2(\ell-j)-1,1+m) \\
= & \sum_{t=0}^{\infty} \sum_{\ell=t}^{n-1+t} \gamma_{n, \ell-t} m^{2 \ell} \frac{\Gamma(s+t)}{\ell ! \Gamma(s+t-j)} \zeta(2 s+2 t-1,1+m) \\
& +\sum_{t=-(n-1)}^{-1} \sum_{\ell=0}^{n-1+t} \gamma_{n, \ell-t} m^{2 \ell} \frac{\Gamma(s+t)}{\ell ! \Gamma(s+t-j)} \zeta(2 s+2 t-1,1+m) .
\end{aligned}
$$

From these formulae, we have

THEOREM 3. The spectral zeta function $\zeta_{P^{n}(C)}$ has a meromorphic continuation on the whole complex plane with at most simple poles at $s=k(k=1,2, \ldots n)$. The residue at $s=k$ is $\gamma_{n, k-1} / 2$.

\section{Spectral zeta functions for quaternionic projective spaces $P^{n}(\boldsymbol{H})$}

Let $P^{n}(\boldsymbol{H})$ denote the quaternionic projective $n$-space with Riemannian metric induced by $2(n+2) \times$ negative of the Cartan-Killing form of $S p(n+1)$. Then every geodesic on $P^{n}(\boldsymbol{H})$ is closed of length $\pi$. By [2], all the eigenvalues of $\Delta_{P^{n}(\boldsymbol{H})}^{0}$ are

$$
k(k+2 n+1), \quad k \geq 0
$$

with multiplicities

$$
\frac{2(k+n+1 / 2)(k+1)\{(k+2) \cdots(k+2 n-1)\}^{2}(k+2 n)}{(2 n+1) !(2 n-1) !} .
$$

So that the spectral zeta function $\zeta_{P^{n}(\boldsymbol{H})}(s)$ of $P^{n}(\boldsymbol{H})$ is of the form:

$$
\zeta_{P^{n}(\boldsymbol{H})}(s)=\sum_{k=1}^{\infty} 2 \frac{(k+n+1 / 2)(k+1)\{(k+2) \cdots(k+2 n-1)\}^{2}(k+2 n)}{(2 n+1) !(2 n-1) !(k(k+2 n+1))^{s}} .
$$

Define the polynomial $H_{n}(x)$ by

$$
\begin{aligned}
H_{n}(x) & =\frac{2}{(2 n+1) !(2 n-1) !}(x+2 n) \prod_{l=1}^{n-1}\left(x+\left(n+\frac{1}{2}\right)^{2}-\left(i-\frac{1}{2}\right)^{2}\right)^{2} \\
& =\sum_{j=0}^{2 n-1} \delta_{n, j} x^{J} .
\end{aligned}
$$


Then we have

$$
\begin{aligned}
\zeta_{P^{n}(\boldsymbol{H})}(s) & =\sum_{k=1}^{\infty} \frac{(k+n+1 / 2) H_{n}(k(k+2 n+1))}{(k(k+2 n+1))^{s}} \\
& =\sum_{j=0}^{2 n-1} \delta_{n, j} \sum_{k=1}^{\infty} \frac{(k+n+1 / 2)}{(k(k+2 n+1))^{s-j}} .
\end{aligned}
$$

Using Corollary 1, we have

$$
\begin{aligned}
\zeta_{P^{n}(\boldsymbol{H})}(s)= & \sum_{j=0}^{2 n-1} \delta_{n, j} \sum_{\ell=0}^{\infty}\left(n+\frac{1}{2}\right)^{2 \ell} \frac{\Gamma(s-j+\ell)}{\ell ! \Gamma(s-j)} \zeta\left(2(s-j)+2 \ell-1, \frac{3}{2}+n\right) \\
= & \sum_{j=0}^{2 n-1} \delta_{n, j} \sum_{\ell=0}^{\infty}\left(n+\frac{1}{2}\right)^{2 \ell} \frac{\Gamma(s-j+\ell)}{\ell ! \gamma(s-j)} \zeta\left(2 s+2(\ell-j)-1, \frac{3}{2}+n\right) \\
= & \sum_{t=0}^{\infty} \sum_{\ell=t}^{2 n-1+t} \delta_{n, \ell-t}\left(n+\frac{1}{2}\right)^{2 \ell} \frac{\Gamma(s+t)}{\ell ! \Gamma(s+t-j)} \zeta\left(2 s+2 t-1, \frac{3}{2}+n\right) \\
& +\sum_{t=-(2 n-1)}^{-1} \sum_{\ell=0}^{2 n-1+t} \delta_{n, \ell-t}\left(n+\frac{1}{2}\right)^{2 \ell} \frac{\Gamma(s+t)}{\ell ! \Gamma(s+t-j)} \zeta\left(2 s+2 t-1, \frac{3}{2}+n\right)
\end{aligned}
$$

From this formula, we have

THEOREM 4. The spectral zeta function $\zeta_{P^{n}(\boldsymbol{H})}(s)$ has a meromorphic continuation on the whole complex plane with at most simple poles at $s=k$ $(k=1,2, \ldots 2 n)$. The residue at $s=k$ is $\delta_{n, k-1} / 2$.

\section{Spectral zeta functions for Cayley projective plane $P^{2}(O)$}

Let $P^{2}(\boldsymbol{O})$ denote the Cayley projective plane with Riemannian metric induced by $18 \times$ negative of the Cartan-Killing form of $F_{4}$. Then every geodesic on $P^{2}(\boldsymbol{O})$ is closed of length $\pi$. By [2], all the eigenvalues of $\Delta_{P^{2}(\boldsymbol{O})}^{0}$ are

$$
k(k+11), \quad k \geq 0,
$$

with their multiplicities

$$
\frac{(2 k+11) 3 !(k+7) !(k+10) !}{11 ! 7 ! k !(k+3) !}
$$

So that the spectral zeta function $\zeta_{P^{2}(\boldsymbol{O})}(s)$ of $P^{2}(\boldsymbol{O})$ is of the form:

$$
\zeta_{P^{2}(\boldsymbol{O})}(s)=\sum_{k=1}^{\infty} \frac{(2 k+11) 3 !(k+7) !(k+10) !}{11 ! 7 ! k !(k+3) !(k(k+11))^{s}} .
$$


Define the polynomial $I(x)$ by

$$
\begin{aligned}
I(x) & =\frac{12}{11 ! 7 !} \prod_{l=1}^{5}\left(x+\left(\frac{11}{2}\right)^{2}-\left(i-\frac{1}{2}\right)^{2}\right) \prod_{l=1}^{2}\left(x+\left(\frac{11}{2}\right)^{2}-\left(i-\frac{1}{2}\right)^{2}\right) \\
& =\frac{12}{11 ! 7 !}(x+10)(x+18)(x+24)(x+28)^{2}(x+30)^{2} \\
& =\sum_{J=0}^{7} \eta_{j} x^{J}
\end{aligned}
$$

Then we have

$$
\begin{aligned}
\zeta_{P^{2}(\boldsymbol{O})} & =\sum_{k=1}^{\infty} \frac{(k+11 / 2) I(k(k+11))}{(k(k+11))^{s}} \\
& =\sum_{j=0}^{7} \eta_{j} \sum_{k=1}^{\infty} \frac{(k+11 / 2)}{(k(k+11))^{s-\jmath}} .
\end{aligned}
$$

Using Corollary 1, we have

$$
\begin{aligned}
\zeta_{P^{n}(\boldsymbol{O})}(s)= & \sum_{j=0}^{7} \eta_{j} \sum_{\ell=0}^{\infty}\left(\frac{11}{2}\right)^{2 \ell} \frac{\Gamma(s-j+\ell)}{\ell ! \Gamma(s-j)} \zeta\left(2(s-j)+2 \ell-1, \frac{13}{2}\right) \\
= & \sum_{j=0}^{7} \eta_{j} \sum_{\ell=0}^{\infty}\left(\frac{11}{2}\right)^{2 \ell} \frac{\Gamma(s-j+\ell)}{\ell ! \Gamma(s-j)} \zeta\left(2 s+2(\ell-j)-1, \frac{13}{2}\right) \\
= & \sum_{t=0}^{\infty} \sum_{\ell=t}^{7+t} \eta_{\ell-t}\left(\frac{11}{2}\right)^{2 \ell} \frac{\Gamma(s+t)}{\ell ! \Gamma(s+t-j)} \zeta\left(2 s+2 t-1, \frac{3}{2}\right) \\
& +\sum_{t=-7}^{-1} \sum_{\ell=0}^{7+t} \eta_{\ell-t}\left(\frac{11}{2}\right)^{2 \ell} \frac{\Gamma(s+t)}{\ell ! \Gamma(s+t-j)} \zeta\left(2 s+2 t-1, \frac{13}{2}\right) .
\end{aligned}
$$

From this formula, we have

THEOREM 5. The spectral zeta function $\zeta_{P^{2}(\boldsymbol{O})}(s)$ has a meromorphic continuation on the whole complex plane with at most simple poles at $s=k$ $(k=1,2, \ldots 8)$. The residue at $s=k$ is $\eta_{k-1} / 2$.

\section{Trivial zeros of $\zeta_{S^{2 m+1}}^{p, \delta}(s)$}

It is well known that the Riemann zeta function $\zeta(s)$ vanishes at even negative integers. In this section, we give analogous results for the spectral zeta function $\zeta_{S^{2 m+1}}^{p, \delta}(s)$. 
THEOREM 6. The spectral zeta function $\zeta_{S^{2 m+1}}^{p, \delta}(s)$ vanishes at negative integers.

Proof. Using the formula (21), we have for a positive integer $k$;

$$
\begin{aligned}
\zeta_{S^{n}}^{p, \delta}(-k)= & \sum_{t=0}^{\infty} \sum_{\ell=t}^{m+t} \alpha_{n, p, \ell-t+1}(m-p)^{2 \ell}(-1)^{\ell}\left(\begin{array}{c}
k-t+\ell \\
k-t
\end{array}\right) \zeta(-2(k-t), 1+m) \\
& +\sum_{t=-m}^{-1} \sum_{\ell=0}^{m+t} \alpha_{n, p, \ell-t+1}(m-p)^{2 \ell}(-1)^{\ell}\left(\begin{array}{c}
k-t+\ell \\
k-t
\end{array}\right) \zeta(-2(k-t), 1+m) \\
= & \sum_{t=0}^{k} \sum_{\ell=t}^{m+t} \alpha_{n, p, \ell-t+1}\left(-(m-p)^{2}\right)^{\ell}\left(\begin{array}{c}
k-t+\ell \\
k-t
\end{array}\right) \zeta(-2(k-t), 1+m) \\
& +\sum_{t=-m}^{-1} \sum_{\ell=0}^{m+t} \alpha_{n, p, \ell-t+1}\left(-(m-p)^{2}\right)^{\ell}\left(\begin{array}{c}
k-t+\ell \\
k-t
\end{array}\right) \zeta(-2(k-t), 1+m) \\
= & \sum_{\ell=k}^{m+k} \alpha_{n, p, \ell-k+1}\left(-(m-p)^{2}\right)^{\ell}\left(\begin{array}{c}
\ell \\
0
\end{array}\right) \zeta(0,1+m) \\
& +\sum_{t=0}^{k-1} \sum_{\ell=t}^{m+t} \alpha_{n, p, \ell-t+1}\left(-(m-p)^{2}\right)^{\ell}\left(\begin{array}{c}
k-t+\ell \\
k-t
\end{array}\right) \zeta(-2(k-t), 1+m) \\
& +\sum_{t=-m}^{-1} \sum_{\ell=0}^{m+t} \alpha_{n, p, \ell-t+1}\left(-(m-p)^{2}\right)^{\ell}\left(\begin{array}{c}
k-t+\ell \\
k-t
\end{array}\right) \zeta(-2(k-t), 1+m) .
\end{aligned}
$$

For the first term, we have

$$
\begin{aligned}
& \sum_{\ell=k}^{m+k} \alpha_{n, p, \ell-k+1}\left(-(m-p)^{2}\right)^{\ell}\left(\begin{array}{l}
\ell \\
0
\end{array}\right) \zeta(0,1+m) \\
& \quad=\sum_{\ell=0}^{m} \alpha_{n, p, \ell+1}\left(-(m-p)^{2}\right)^{\ell+k} \zeta(0,1+m) \\
& \quad=\zeta(0,1+m)\left(-(m-p)^{2}\right)^{k-1} \sum_{\ell=0}^{m} \alpha_{n, p, \ell+1}\left(-(m-p)^{2}\right)^{\ell+1} \\
& =\zeta(0,1+m)\left(-(m-p)^{2}\right)^{k-1} F_{n, p}\left(-(m-p)^{2}\right) \\
& =0 .
\end{aligned}
$$


Thus, we have

$$
\begin{aligned}
& \zeta_{S^{n}}^{p, \delta}(-k) \\
& =\sum_{t=0}^{k-1} \sum_{\ell=t}^{m+t} \alpha_{n, p, \ell-t+1}\left(-(m-p)^{2}\right)^{\ell}\left(\begin{array}{c}
k-t+\ell \\
k-t
\end{array}\right) \zeta(-2(k-t), 1+m) \\
& +\sum_{t=-m}^{-1} \sum_{\ell=0}^{m+t} \alpha_{n, p, \ell-t+1}\left(-(m-p)^{2}\right)^{\ell}\left(\begin{array}{c}
k-t+\ell \\
k-t
\end{array}\right) \zeta(-2(k-t), 1+m) \\
& =\sum_{\ell=1}^{m+1} \alpha_{n, p, \ell} \sum_{t=0}^{k-1}\left(-(m-p)^{2}\right)^{\ell+t-1}\left(\begin{array}{c}
k+\ell-1 \\
k-t
\end{array}\right) \zeta(-2(k-t), 1+m) \\
& +\sum_{\ell=1}^{m+1} \alpha_{n, p, \ell} \sum_{t=-(\ell-1)}^{-1}\left(-(m-p)^{2}\right)^{\ell+t-1}\left(\begin{array}{c}
k+\ell-1 \\
k-t
\end{array}\right) \zeta(-2(k-t), 1+m) \\
& =-\sum_{\ell=1}^{m+1} \alpha_{n, p, \ell} \sum_{t=0}^{k-1}\left(-(m-p)^{2}\right)^{\ell+t-1}\left(\begin{array}{c}
k+\ell-1 \\
k-t
\end{array}\right) \sum_{t=1}^{m} i^{2(k-t)} \\
& -\sum_{\ell=1}^{m+1} \alpha_{n, p, \ell} \sum_{t=-(\ell-1)}^{-1}\left(-(m-p)^{2}\right)^{\ell+t-1}\left(\begin{array}{c}
k+\ell-1 \\
k-t
\end{array}\right) \sum_{t=1}^{m} i^{2(k-t)} \\
& =-\sum_{l=1}^{m} \sum_{\ell=1}^{m+1} \alpha_{n, p, \ell} \sum_{t=0}^{k-1}\left(-(m-p)^{2}\right)^{\ell+t-1}\left(\begin{array}{c}
k+\ell-1 \\
k-t
\end{array}\right) i^{2(k-t)} \\
& -\sum_{l=1}^{m} \sum_{\ell=1}^{m+1} \alpha_{n, p, \ell} \sum_{t=-(\ell-1)}^{-1}\left(-(m-p)^{2}\right)^{\ell+t-1}\left(\begin{array}{c}
k+\ell-1 \\
k-t
\end{array}\right) i^{2(k-t)} \\
& =-\sum_{l=1}^{m} \sum_{\ell=1}^{m+1} \alpha_{n, p, \ell} \sum_{t=-(\ell-1)}^{k-1}\left(-(m-p)^{2}\right)^{\ell+t-1}\left(\begin{array}{c}
k+\ell-1 \\
k-t
\end{array}\right) i^{2(k-t)} \\
& =-\sum_{\ell=1}^{m} \sum_{\ell=1}^{m+1} \alpha_{n, p, \ell} \sum_{t=0}^{k+\ell-2}\left(-(m-p)^{2}\right)^{t}\left(\begin{array}{c}
k+\ell-1 \\
k+\ell-1-t
\end{array}\right) i^{2(k+\ell-1-t)} \\
& =-\sum_{\ell=1}^{m} \sum_{\ell=1}^{m+1} \alpha_{n, p, \ell} \sum_{t=0}^{k+\ell-1}\left(\begin{array}{c}
k+\ell-1 \\
k+\ell-1-t
\end{array}\right)\left(-(m-p)^{2}\right)^{t} i^{2(k+\ell-1-t)} \\
& +\sum_{i=1}^{m} \sum_{\ell=1}^{m+1} \alpha_{n, p, \ell}\left(\begin{array}{c}
k+\ell-1 \\
0
\end{array}\right)\left(-(m-p)^{2}\right)^{k+\ell-1} i^{0}
\end{aligned}
$$




$$
\begin{aligned}
= & -\sum_{l=1}^{m} \sum_{\ell=1}^{m+1} \alpha_{n, p, \ell}\left(-(m-p)^{2}+i^{2}\right)^{k+\ell-1}+\sum_{l=1}^{m} \sum_{\ell=1}^{m+1} \alpha_{n, p, \ell}\left(-(m-p)^{2}\right)^{k+\ell-1} \\
= & \sum_{l=1}^{m}-\left(-(m-p)^{2}+i^{2}\right)^{k-1} F_{n, p}\left(-(m-p)^{2}+i^{2}\right) \\
& +\left(-(m-p)^{2}\right)^{k-1} \sum_{l=1}^{m} F_{n, p}\left(-(m-p)^{2}\right) \\
= & 0 .
\end{aligned}
$$

\section{REFERENCES}

[1] M. Berger, P Gauduchon and E. Mazet, Le Spectre d'une Variété Riemannianne, Lecture Notes in Math., 194, Sprınger-Verlag, Berlin, 1971.

[2] R. S. CAHN AND J. A. WOlF, Zeta functions and their asymptotic expansions for compact symmetric spaces of rank one, Comment. Math. Helv., 51 (1976), 1-21.

[3] E. Carletti and G. Monti Bragardin, On Dirichlet series associated with polynomials, Proc. Amer. Math. Soc., 121 (1994), 33-37

[4] E. Carletti and G. Monti Bragardin, On Minakshisundaram-Pleijel zeta functions of spheres, Proc. Amer. Math. Soc., 122 (1994), 993-1001.

[ 5] M. EIE, On a Dirichlet series associated with Polynomial, Proc. Amer. Math. Soc., 110 (1990), 583-590.

[6] P GiLkeY, Invariance Theory, the Heat Equation, and the Atyah-Singer Index Theorem, 2nd ed., CRC Press, 1994.

[7] A. IKEDA AND Y TANigUCHI, Spectra and eigenforms of the Laplacian on $S^{n}$ and $P^{n}(C)$, Osaka J. Math., 15 (1978), 515-546.

[8] A. IKEDA, Zeta functions for differential forms on standard spheres and their asymptotıc expansions, Bull. Fac. School Edu. Hiroshıma Unıv. Part II, 11 (1988), 31-40.

[9] S. Minakshisundaram and A. Pleijel, Some properties of the eigenvalues of the Laplace operator on Riemannian manifolds, Canad. J. Math., 1 (1949), 242-256.

[10] S. Minakshisundaram, Zeta functions on the spheres, J. Indian Math. Soc., 13 (1949), $41-$ 48.

[11] R. SunAda, Fundamental groups and Laplacian, Kinokuniya, Tokyo, 1988 (in Japanese).

[12] E. T. Whittaker and G. N. Watson, Modern Analysis, Cambridge Univ. Press., London, 1958.

DEPARTMENT OF MATHEMATICS

FACULTY OF EDUCATION

OKaYAMa UNIVERSITY

OKAYAMA 700-8530, JAPAN 